\title{
Escaping Flatland: three-dimensional kinematics and hydrodynamics of median fins in fishes
}

\section{Citation}

Tytell, E. D., E. M. Standen, and G. V. Lauder. 2008. Escaping Flatland: Three-Dimensional Kinematics and Hydrodynamics of Median Fins in Fishes. Journal of Experimental Biology 211, no. 2: 187-195. doi:10.1242/jeb.008128.

\section{Published Version}

doi:http:10.1242/jeb.008128

\section{Permanent link}

http://nrs.harvard.edu/urn-3:HUL.InstRepos:30510342

\section{Terms of Use}

This article was downloaded from Harvard University's DASH repository, and is made available under the terms and conditions applicable to Other Posted Material, as set forth at http:// nrs.harvard.edu/urn-3:HUL.InstRepos:dash.current.terms-of-use\#LAA

\section{Share Your Story}

The Harvard community has made this article openly available.

Please share how this access benefits you. Submit a story.

\section{Accessibility}




\title{
Escaping Flatland: three-dimensional kinematics and hydrodynamics of median fins in fishes
}

\author{
Eric D. Tytell ${ }^{1, *}$, Emily M. Standen ${ }^{2}$ and George V. Lauder ${ }^{2}$ \\ ${ }^{1}$ Department of Biology, University of Maryland, College Park, MD 20742, USA and ${ }^{2}$ Department of Organismic and Evolutionary \\ Biology, Harvard University, Cambridge, MA 02138, USA \\ *Author for correspondence (e-mail: tytell@umd.edu)
}

Accepted 24 May 2007

\begin{abstract}
Summary
Fish swimming has often been simplified into the motions of a two-dimensional slice through the horizontal midline, as though fishes live in a flat world devoid of a third dimension. While fish bodies do undulate primarily horizontally, this motion has important three-dimensional components, and fish fins can move in a complex three-dimensional manner. Recent results suggest that an understanding of the three-dimensional body shape and fin motions is vital for explaining the mechanics of swimming, and that two-dimensional representations of fish locomotion are misleading. In this study, we first examine axial swimming from the twodimensional viewpoint, detailing the limitations of this view. Then we present data on the kinematics and hydrodynamics of the dorsal fin, the anal fin and the caudal fin during steady swimming and maneuvering in brook trout, Salvelinus fontinalis, bluegill sunfish, Lepomis macrochirus, and yellow perch, Perca flavescens. These fishes actively move the dorsal and anal fins during swimming, resulting in curvature along both anterio-posterior and dorso-ventral axes. The momentum imparted to the fluid by these fins comprises a substantial portion of total swimming force, adding to thrust and contributing to roll stability. While swimming, the caudal fin also actively curves dorso-ventrally, producing vortices separately from both its upper and lower lobes. This functional separation of the lobes may allow additional control of three-dimensional orientation, but probably reduces swimming efficiency. In contrast, fish may boost the caudal fin's efficiency by taking advantage of the flow from the dorsal and anal fins as it interacts with the flow around the caudal fin itself. During maneuvering, fish readily use their fins outside of the normal planes of motion. For example, the dorsal fin can flick laterally, orienting its surface perpendicular to the body, to help in turning and braking. These data demonstrate that, while fish do move primarily in the horizontal plane, neither their bodies nor their motions can accurately be simplified in a two-dimensional representation. To begin to appreciate the functional consequences of the diversity of fish body shapes and locomotor strategies, one must escape Flatland to examine all three dimensions.
\end{abstract}

Key words: fish, locomotion, hydrodynamics, flow, kinematics, dorsal fin, anal fin, caudal fin.

\section{Introduction}

'I exhorted all my hearers to divest themselves of prejudice and to become believers in the Third Dimension.'

E. A. Abbott, Flatland: A Romance of Many Dimensions, 1899

In the classic book Flatland, E. A. Abbott describes a world of two dimensions, a world in which the inhabitants live in ignorance of the third dimension, a world in which it is impossible to distinguish circles from squares, and squares from pentagons everything looks like a line in the two-dimensional world of Flatland (Abbott, 1899). Then, a disruptive Sphere enters to make inhabitants aware of a third dimension, causing consternation and ultimately sending the narrator to jail. The Flatland of Abbott's book is an appropriate metaphor for much of the current literature on undulatory locomotion in fishes, which is largely devoted to analyses of fish swimming in the horizontal (two-dimensional, 2D) plane. This 2D world has, so far, been an appropriate place to conduct our studies of fish locomotion: fewer cameras are needed to acquire data, analyses are simpler, and theoretical models can be generated more easily (e.g. Schultz and Webb, 2002).

But fish are three-dimensional (3D), with prominent median and paired fins that project into the water, and with clearly defined edges that demark the dorsal, ventral and caudal body surface. Although thinking in three dimensions may complicate our lives, it is nonetheless a critical next step that experimental and computational analyses of fish undulatory locomotion are only now starting to grapple with. Like the residents of Flatland, we must learn to deal with the 3D world.

The 3D nature of fish functional design is clearly seen in the enormous diversity of body shapes and swimming modes in fishes, but the precise advantage of one shape or mode over another is less clear. Generalizations from hydrodynamic theories combined with observations of typical swimming behavior have led to much speculation on the adaptations of certain morphologies to different situations (Lighthill, 1975; Marshall, 1971). Tunas, for example, are highly specialized in many ways, many of which are probably adaptations to their active, pelagic lifestyle (Block and Stevens, 2001). However, eels, which have a different body shape and swimming mode from tunas, also migrate thousands of kilometers 
without feeding (van Ginneken et al., 2005). Presumably, the different body shapes and swimming modes of these fishes confer different advantages, but the exact functional benefits of their morphologies remains unclear.

In Flatland, most of these morphological differences are not evident. In horizontal cross-section, even eels and tunas are not unlike each other: they both have streamlined bodies with highaspect ratios, and they propel themselves with an undulatory wave that increases in amplitude near the tail. This similarity among fishes was a boon for classic theories of fish swimming (e.g. Gray, 1933; Lighthill, 1960; Taylor, 1952; Wu, 1971), because a 2D undulating plate or cylinder serves as a good initial approximation of most fishes. The use of this simplification allowed much progress in understanding the basic mechanisms of fish swimming.

In recent years, the empirical understanding of fish swimming has advanced beyond the classic theories to produce a general description of flow patterns during fish swimming. These advances have been achieved mostly through the technique of particle image velocimetry (PIV) (Willert and Gharib, 1991). PIV has established the fluid flow patterns near the body (Anderson et al., 2001) and in the wake of many fishes (Drucker and Lauder, 1999; Müller et al., 2001; Müller et al., 1997; Nauen and Lauder, 2002a; Nauen and Lauder, 2002b; Tytell, 2004; Tytell and Lauder, 2004). The basic propulsive flow structure is a set of alternating jets, oriented laterally and posteriorly. The wake structure behind eels seems to be somewhat different from this basic pattern (Müller et al., 2001; Tytell and Lauder, 2004), which we will argue below is a sign of the importance of the 3D eel body shape. Even the wake of eels, though, is not so different as to indicate dramatic differences in propulsive mechanisms from those of other fishes. In general, the overall picture that has emerged is one of consistency, from eels to mackerel.

The consistency may be, in part, a result of the 2D approximation. PIV is a fundamentally 2D technique: it only permits measurements of flow velocities in a plane. The underlying assumption of most PIV studies of fish swimming has been that the flow in a plane along the horizontal midline accurately captures the 3D flow around the fish. Some studies have used multiple orthogonal planes to establish a better approximation of the 3D flow structure (Drucker and Lauder, 1999; Nauen and Lauder, 2002a), but even these make the assumption that flow in a midline plane (whether horizontal or vertical) captures the structure of flow in parallel planes offset from the midline. However, Tytell's results in bluegill sunfish suggest that flow in a horizontal plane at the level of the dorsal or ventral tips of the caudal fin may be quite different from flow in the horizontal midline (Tytell, 2006).

Our view is that the current understanding of undulatory propulsion, from empirical, theoretical and computational standpoints, has progressed to the point that 3D effects should no longer be neglected. There are two components to these effects: (1) the static 3D body shape and (2) the dynamic 3D motion of the body. As we begin to include these effects, we return to the roots of studies of fish swimming, by connecting hydrodynamics to functional morphology. With a 3D perspective, we can start to address questions of adaptation in a quantitative way, examining the relationship of swimming performance to $3 \mathrm{D}$ body shape and kinematics. Also, describing how maneuvers are accomplished requires a 3D analysis, due to the complex and often asymmetric positions and kinematics of fins during maneuvers.

In this paper, we first describe recent data on the $3 \mathrm{D}$ character of the swimming motion, both in steady swimming and during maneuvering. We focus on the dorsal and anal fins, which are often neglected entirely in the 2D approximation (but not in all models) (see Weihs, 1972) but, for many fishes, constitute nearly as much fin area as the caudal fin and have their own complex 3D motion. Then, we describe evidence that the $3 \mathrm{D}$ shape and motion of the median fins is important to the overall flow structure around the fish. The importance of 3D effects is seen in three ways: (1) the effect of the 3D motion itself, which we illustrate by focusing on the caudal fin; (2) the effect of the dorsal and anal fins, as an indication of the importance of 3D body shape; and (3) the interaction between flows from different fins.

\section{Problems with the 2D approximation}

As an example of the problems with the 2D approximation, consider a classic distinction in modes of fish swimming: the anguilliform mode, used by eels and sharks primarily, and the carangiform mode, used by most other fishes (Breder, 1926). Gray's early studies of eels (Gray, 1933) indicated erroneously that eels swim very differently from other fishes (Lauder and Tytell, 2004). In contrast to Gray's results, recent close examination of the midline kinematics of these two modes has shown that the differences between them are quite subtle (Fig. 1) (Gillis, 1998; Lauder and Tytell, 2006), particularly at the low swimming speeds commonly used for long distance swimming (van Ginneken et al., 2005).

Despite the similarity of the midlines' movements, studies of the flow in the wakes of anguilliform and carangiform swimmers showed different flow patterns (e.g. Müller et al., 2001; Nauen and Lauder, 2002a; Tytell and Lauder, 2004). This led Lauder and Tytell to conclude that differences in 3D body shape (Fig. 1A,C) must account for the differences in wake structure (Lauder and Tytell, 2006).

\section{D body shapes}

To some extent, classic 2D theories can account for differences in body shapes among fishes. Most theories, including Lighthill's

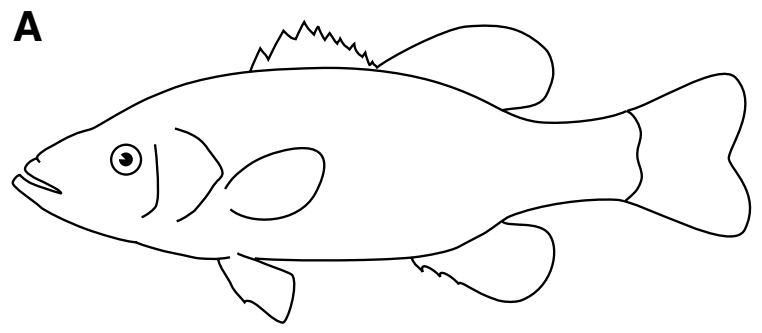

B Bass, $0.7 L^{-1}$
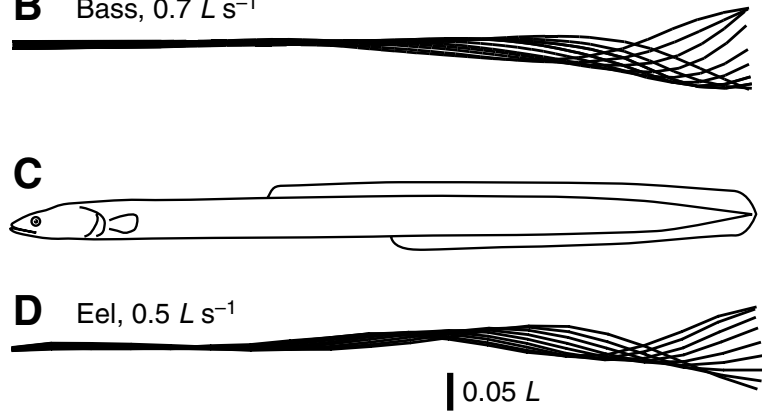

Fig. 1. Comparison of three-dimensional (3D) body shape and midline kinematics for a bass (Micropterus salmoides) and an eel (Anguilla rostrata). (A,C) Line drawings of the lateral profiles. $(B, D)$ Midline tracings at equally spaced intervals of time during slow swimming at 0.7 lengths s $\mathrm{s}^{-1}$ $\left(L \mathrm{~s}^{-1}\right)$ for the bass (modified from Jayne and Lauder, 1995) and $0.5 \mathrm{~L} \mathrm{~s}^{-1}$ for the eel (E.D.T., unpublished data). Scale bar is valid for both panels. 


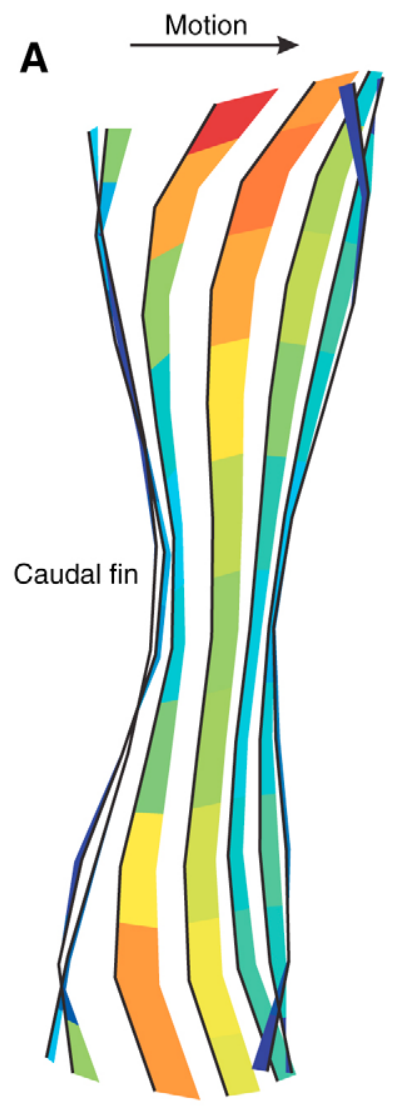

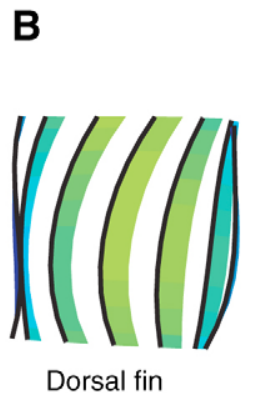

15

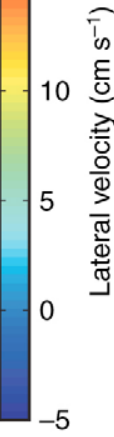

$5 \mathrm{~mm}$

$10 \mathrm{~cm} \mathrm{~s}^{-1}$ elongated body theory, include a term that expresses the dorsoventral height of the fish (Lighthill, 1971). To satisfy the assumptions in the theories, however, this term cannot vary too quickly along the body length. In other words, fishes like tunas and mackerels with pronounced narrow peduncles and broad tails cannot be approximated in this way (Lighthill, 1971); instead, Lighthill developed an alternative 2D theory that approximated just the tail (Lighthill, 1970).

Several recent lines of evidence suggest that the body shape of many fishes may produce 3D flow patterns that do not conform to the assumptions in these 2D theories. Experimentally, Nauen and Lauder found that flow around the caudal peduncle and finlets of mackerel converged towards the horizontal midline and wrapped above and below the peduncle (Nauen and Lauder, 2001), a combination that cannot be treated well with a 2D approximation like Wu's waving plate or elongated body theory (Lighthill, 1971; Wu, 1971). Computational results supported the same conclusion for an approximation of a giant danio morphology (Zhu et al., 2002). Additionally, both elongated body theory (Lighthill, 1971) and a resistive model (after Taylor, 1952) were compared with PIV estimates of lateral forces in the wake of an eel. Neither model individually, nor the combination of the two, could fully account for the forces estimated experimentally (Tytell, 2004).

\section{D kinematics}

Not only are fish body shapes 3D, but the fins typically move in a 3D manner. Median fins actively oscillate, bend, curve and flick in all directions under the control of fin ray musculature. By erecting and depressing their rays, fish can control both the shape of their fins and the how much fin area is exposed to the flow.

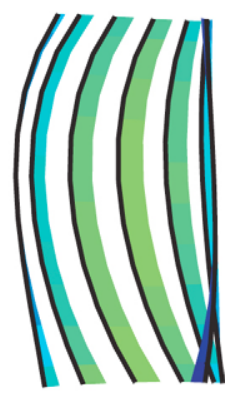

Anal fin

Fig. 2. Tips of the caudal, dorsal and anal fins in bluegill sunfish cup actively into the flow. The motion of fins through half a tail beat from left to right in two transverse planes is presented. Each panel shows seven tracings of the posterior margins of the fins, spaced equally in time, as seen from behind the fish. The color and thickness of the bar indicate the lateral velocity of a particular segment of the fin. The beat frequency for each fin is the same, $2.4 \mathrm{~Hz}$. (A) Caudal fin kinematics. (B) Dorsal and anal fin kinematics. Inset in A shows position of the two planes. Scales are the same for A and B (from Tytell, 2006).

Fig. 2 illustrates control of fin shape. Bluegill sunfish move the dorsal and ventral edges of the caudal fin further and faster than the middle (Fig. 2A) (Lauder, 2000; Tytell, 2006), resulting in a 'cupping' motion into the flow. Bainbridge reported similar results for the caudal fin of dace (Leuciscus leuciscus) (Bainbridge, 1963). The dorsal and anal fins also have a similar cupping motion (Fig. 2B) (Standen and Lauder, 2005; Tytell, 2006).

Fig. 3 shows data on changes in the exposed area of fins in bluegill sunfish. Bluegill sunfish, trout and yellow perch all

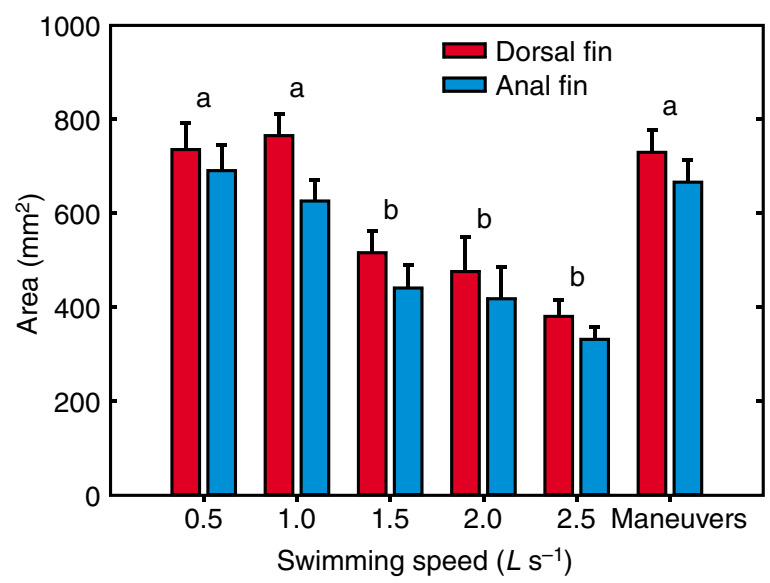

Fig. 3. Fin area in bluegill sunfish changes at different swimming speeds due to the elevation or depression of the fin rays. Bars show mean fin surface area at the time of maximum excursion and error bars represent s.e.m. Fin area at swimming speeds denoted with letter a differ significantly from those denoted by letter b (from Standen and Lauder, 2005). 

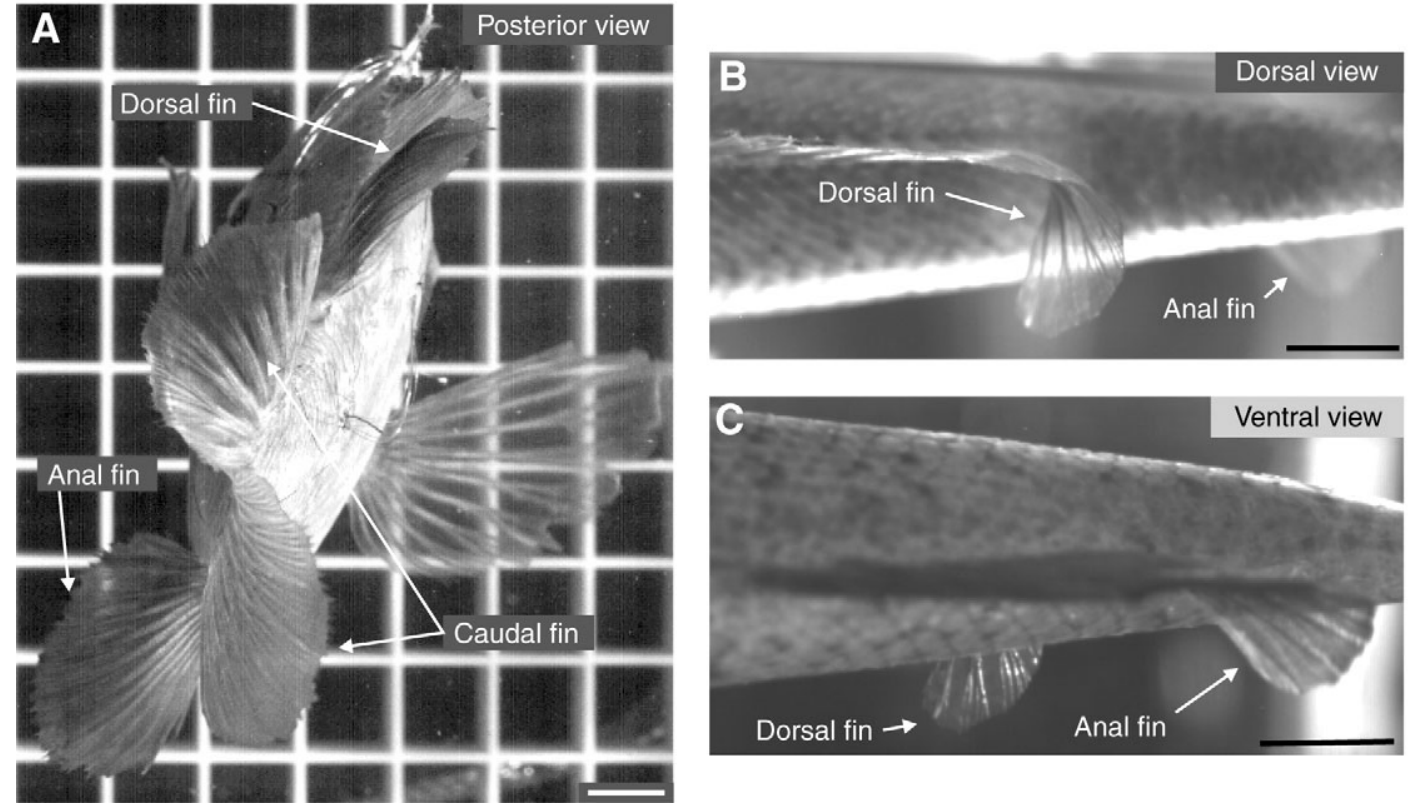

Fig. 4. Posterior, dorsal and ventral views of maneuvering behaviors in bluegill sunfish. (A) Posterior view during a braking maneuver. Note the asymmetry of the caudal fin. The caudal edge of the dorsal fin is curled forward into the flow. Image courtesy of Brooke Flammang (unpublished data). $(B, C)$ Dorsal and ventral views of a right turning maneuver. Both dorsal and anal fins show displacement of their trailing edges during this maneuver. Curvature of the fin surfaces is large, but the actual bending of each individual fin ray is small. Scale bars throughout are $1 \mathrm{~cm}$ (from Standen and Lauder, 2005). decrease dorsal and anal fin area with increasing swimming speed (Fig. 3) (Drucker and Lauder, 2001; Drucker and Lauder, 2005; Standen and Lauder, 2005). Reducing surface area affects the function of the fin in two ways: (1) by reducing the amount of drag on the fin, mostly from skin friction, and (2) by reducing the fin area available to produce useful forces. Such changes in the functional area of fins are not easily incorporated into 2D kinematic studies of fish swimming.

The dynamic and 3D nature of the median fins is most dramatically seen during maneuvers (Fig. 4). During braking and turning maneuvers, dorsal and anal fins are used asymmetrically and sometimes asynchronously to produce forces that change body
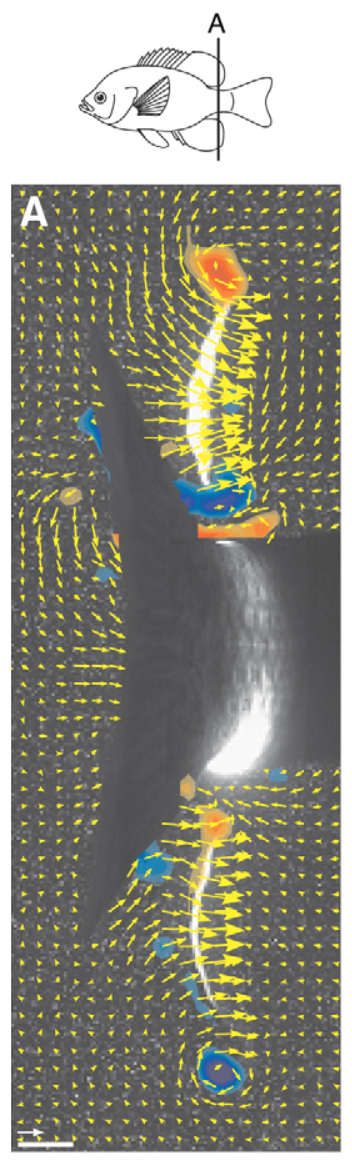
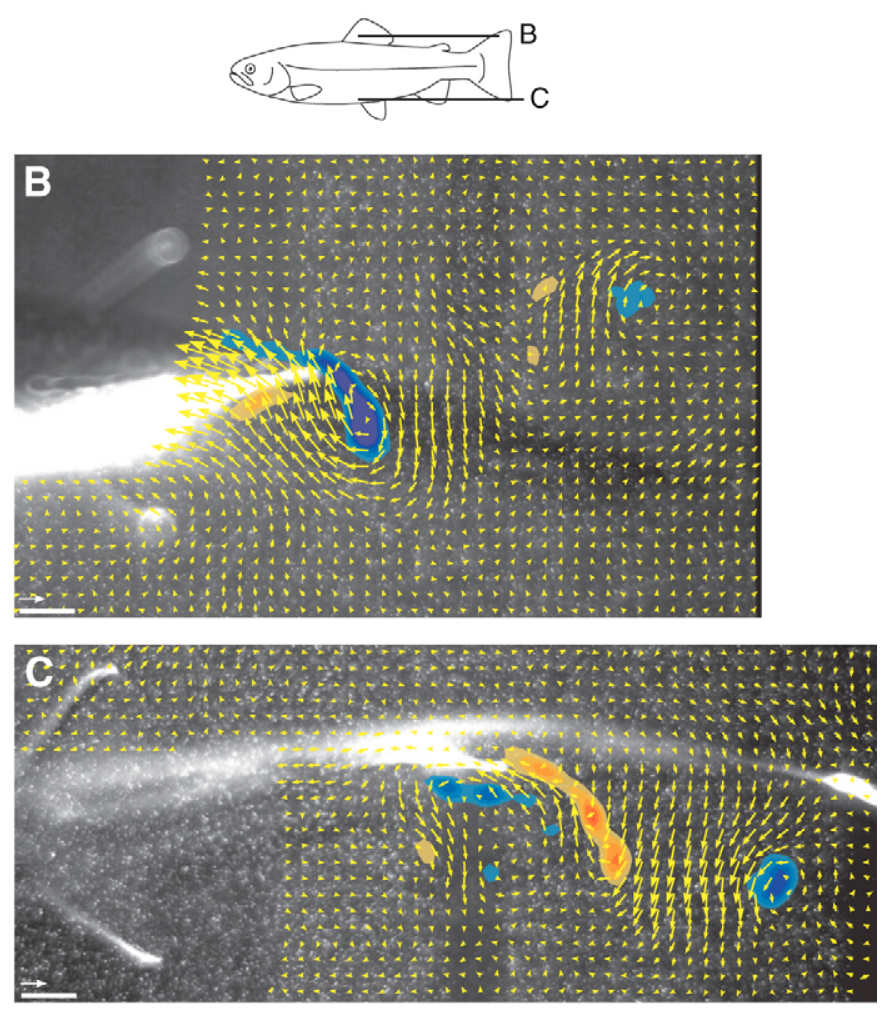

$-40-35-30-25-20-15 \quad 15 \quad 20 \quad 25 \quad 30 \quad 35 \quad 40$

Vorticity $\left(\mathrm{s}^{-1}\right)$
Fig. 5. Representative flow fields near the dorsal and anal fins during steady swimming. Yellow arrows indicate flow velocity; colored contours show vorticity. Schematic diagrams above the plots indicate the approximate position and orientation of the light sheets.

(A) Flow in a transverse plane near the posterior edges of the dorsal and anal fins of bluegill sunfish swimming at $1.2 \mathrm{~L} \mathrm{~s}^{-1}$. Vortices are shed along each dorsal and ventral edge of both the dorsal and anal fins. The caudal fin obscures some of the view (from Tytell, 2006). (B,C) Flow in horizontal planes at the level of the dorsal fin (B) and the anal fin (C) of trout swimming at $0.5 \mathrm{~L} \mathrm{~s}^{-1}$. The pelvic fins are visible on the left side of both images, and $\mathrm{C}$ shows both the anal fin (center) and some of the of the caudal fin (right) (from Standen and Lauder, 2007). Scale bars throughout are $5 \mathrm{~mm}$ and scale vectors are $5 \mathrm{~cm} \mathrm{~s}^{-1}$. 
position and orientation. During braking, bluegill sunfish often extend dorsal and anal fins to opposite sides of the body. In addition, the tail can generate extremely asymmetrical shapes, with the dorsal and ventral lobes forming an s-curve with the lobes extending to opposite sides from each other and their respective anterior median fins (Fig. 4A). A braking fin formation such as is seen in Fig. 4A creates a large symmetrical increase in drag from the median fins. In addition, during turning maneuvers, the dorsal and anal fins often display near $90^{\circ}$ bends in their surface (Fig. 4B,C).

\section{Importance of the 3D view}

In the above sections, we have argued that both fishes and the swimming motion itself are inescapably $3 \mathrm{D}$, particularly during unsteady behaviors like maneuvering. But how important is the third dimension? Is it really necessary to add the extra complexity that a 3D analysis brings? Below, we present three lines of argument that 3D body shape and kinematics must be considered to further the current understanding of fish swimming.

First, the kinematics themselves, even during steady swimming, have a $3 \mathrm{D}$ character. We claim that the $3 \mathrm{D}$ motion probably affects the amount of force produced and the efficiency by which the tail transfers momentum to the fluid. In dace (Bainbridge, 1963) and bluegill sunfish (Lauder, 2000; Tytell, 2006), the dorsal and ventral tips of the caudal fin actively cup into the flow as the fin moves from side to side (Fig. 2). The tips move about twice as far as the horizontal midline in the same amount of time; therefore, they move approximately twice as fast. In general, fluid dynamic forces scale as velocity squared; thus, force could vary along the height of the caudal fin by as much as four times. To correctly estimate force, one must therefore look at the 3D motion of the fin, not the 2D motion of any individual horizontal slice. Additionally, the cupping motion of the fin distorts the vortex ring shed into the wake into a complex, non-circular form (Tytell, 2006). A circular loop, in contrast, is the most efficient way of transferring momentum to the fluid (Lighthill, 1970). Thus, the independent control of the dorsal and ventral lobes of the caudal fin, which is important for maneuvering (e.g. as seen in Fig. 4A), may reduce the efficiency of steady swimming for bluegill sunfish.

Second, several recent studies (Drucker and Lauder, 2005; Standen and Lauder, 2007; Tytell, 2006) indicate that the dorsal and anal fins, which are typically neglected in 2D approximations (but see Weihs, 1972), actively produce forces that are a sizeable
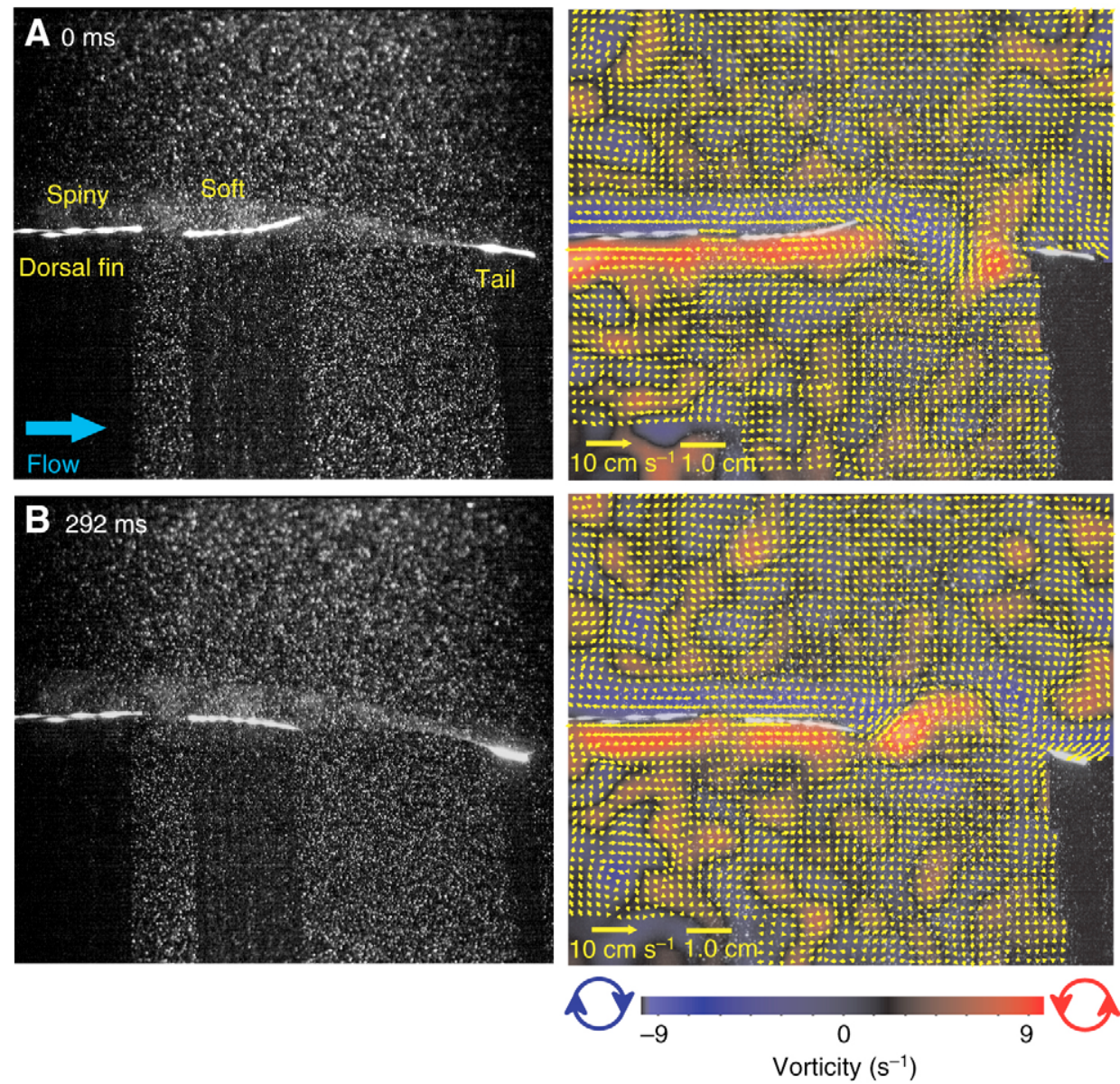

Fig. 6. Hydrodynamic function of the dorsal fin in yellow perch (Perca flavescens, $18.0 \mathrm{~cm}$ total length, $L$ ) to illustrate fin positions (left panels) and wake flows (right panels) during steady swimming at $0.5 \mathrm{~L} \mathrm{~s}^{-1}$. The spiny and soft dorsal fins and the dorsal lobe of the caudal fin have been illuminated with a horizontal laser light sheet for particle image velocimetry. (A) Fin positions and wake flows at a time when the soft dorsal fin has reached maximum excursion to the right; (B) Fin positions 292 ms later when the soft dorsal fin is at its maximal excursion to the left side of the perch. Note the shear layer resulting from the spiny and soft dorsal fins, evident as the elongated regions of red and blue vorticity in the right-hand panels. The soft dorsal fin also sheds a drag wake with regions of vorticity visible in the gap between the soft dorsal fin and the caudal fin. Images on the left have been contrastenhanced to better reveal the laser-illuminated particles. Shadows where vectors were not calculated represent regions where the laser light sheet was blocked by the fish body or fins. 

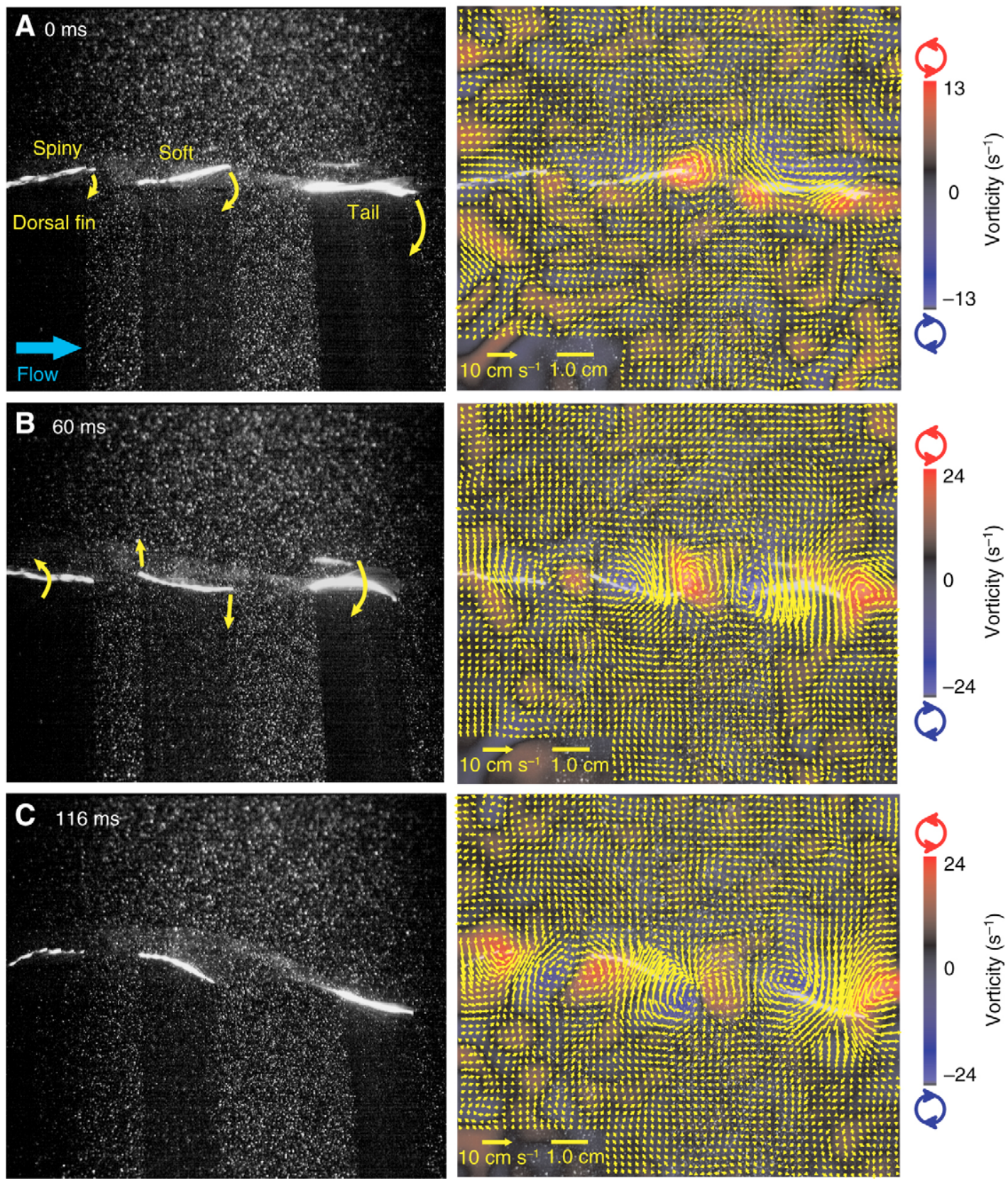

Fig. 7. Hydrodynamic function of the dorsal fin in yellow perch (Perca flavescens, $18.0 \mathrm{~cm}$ total length, $L$ ) to illustrate wake flows during a low-speed yawing turn elicited during steady swimming at $0.5 \mathrm{~L} \mathrm{~s}^{-1}$. A horizontal laser light sheet is illuminating both the spiny and soft portions of the dorsal fin, as well as the dorsal lobe of the caudal fin. Fin positions and wake flows are illustrated for the early, middle and late stages of the turn. The yellow arrows in the left-hand panels show the direction of fin surface motion from one panel to the next. Initial wake flows during the turn are generated by the soft dorsal fin (A). As the turn develops, both the soft dorsal and the caudal fin have generated distinct momentum jets, which result in a yawing turn of the perch body (B). At the end of the turn, each of the three fins has shed a distinct vortex ring (C). Images on the left have been contrast-enhanced to better reveal the laser-illuminated particles.

contribution to the forces produced by the fish as a whole. These studies use circulation, a measure of the strength of a vortex, as an estimate of force production. Fig. 5 shows vortices shed by the dorsal and anal fins in trout and bluegill sunfish. In bluegill sunfish, the dorsal and anal fins shed vortices (Fig. 5A) with circulations that are statistically indistinguishable from those produced by the caudal fin, indicating that the total force from the anterior median fins is comparable to that from the tail itself (Tytell, 2006). Recordings from the inclinator muscles that move the dorsal fin in sunfish (Jayne et al., 1996) show that bluegill sunfish actively move the soft dorsal fin: it is not oscillating passively with the body. The dorsal fin wake has both lateral (side) and thrust (posterior) components, with a ratio of side to posterior force of approximately 2:1 for fish swimming at 1.0 lengths $\mathrm{s}^{-1}\left(\mathrm{~L} \mathrm{~s}^{-1}\right)$ (Drucker and Lauder, 2001). The side forces produced by the dorsal fin may be important in maintaining stability, while the thrust component adds to locomotor thrust forces generated by the tail and the anal fin. In trout, also, the dorsal and anal fins produce substantial vortices (Fig. 5B,C) (Drucker and Lauder, 2005; Standen and Lauder, 2007). These vortices result in jets with a large lateral component, 


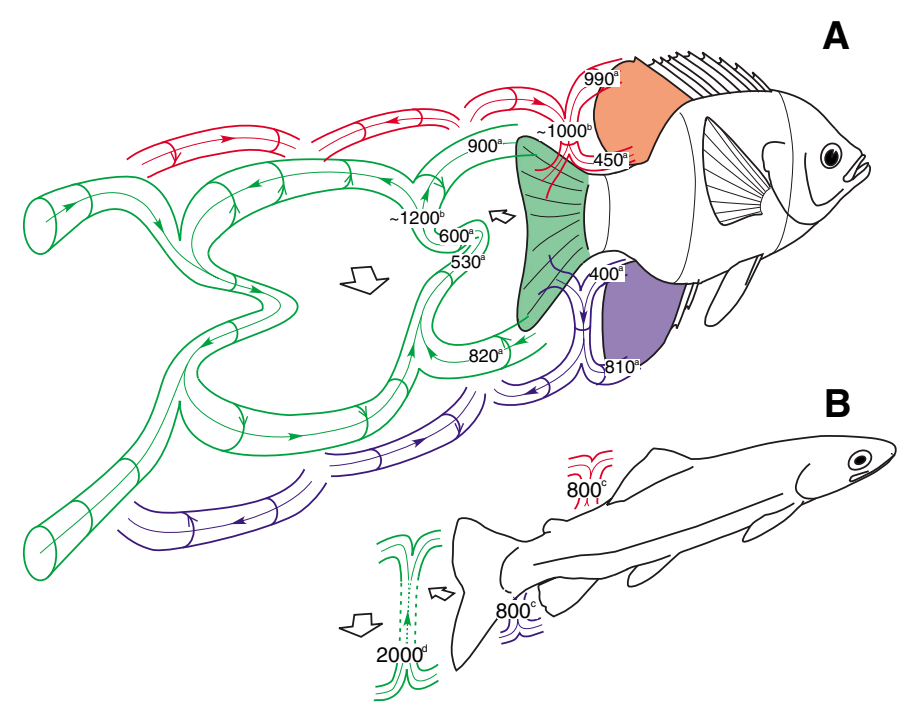

indicating that their effect is more on roll stability of the fish, rather than propulsion (Drucker and Lauder, 2005; Standen and Lauder, 2007). Because both fins are located above and below the rolling axis of trout, the lateral forces from these fins result in rolling torque production during swimming. In brook trout, these torques appear to balance (Standen and Lauder, 2007), helping to maintain the fish in an upright orientation. Similar dorsal fin momentum jets are also observed in yellow perch (Figs 6,7).

The measurements from Fig. 5 can be integrated to produce a 3D hypothesis for the vortex flow around fishes (Fig. 8). The most complete data set exists for bluegill sunfish (Fig. 8A), but there are also substantial data from horizontal planes for trout (Fig. 8B). Circulation estimates for fishes swimming at 1.2 and $0.5 \mathrm{~L} \mathrm{~s}^{-1}$ are superimposed on the vortex diagram, as an indication of the relative importance of different vortices.
Fig. 8. Vortex filament model showing vortices near steadily swimming bluegill sunfish and brook trout. Numbers indicate vortex circulation (in $\mathrm{mm}^{2} \mathrm{~s}^{-1}$ ), to evaluate the relative vortex strengths, and the superscript letter identifies the source (see below). (A) 3D vortex structure for bluegill sunfish swimming at about $1.2 \mathrm{~L} \mathrm{~s}^{-1}$. Gaps in the dorsal and anal fin vortices in the wake indicate regions where the vortices should link up to the caudal fin vortices, but for which there are too few data to identify the topology of the filament. (B) Experimentally observed vortices for the dorsal, anal and caudal fins of trout swimming at $0.5 \mathrm{~L} \mathrm{~s}^{-1}$. Dotted lines in the caudal fin vortices indicate a region that might have a deformation, like in bluegill sunfish, but for which there are not appropriate data for any definitive conclusions. Sources of circulation data: a (Tytell, 2006); ${ }^{b}$ (Drucker and Lauder, 2001) (estimated from published figures); ${ }^{\circ}($ Standen and Lauder, 2007); ${ }^{\mathrm{d}}$ (E.M.S. and G.V.L., unpublished data).
Finally, the vortices shown diagrammatically in Fig. 8 do not occur in isolation; they influence each other and the flow around the body of the fish. Drucker and Lauder showed that the dorsal fin of bluegill sunfish produces a strong wake that moves posteriorly to encounter the tail as it beats from side to side (Drucker and Lauder, 2001). The dorsal fin wake can directly alter thrust generated by the tail as a result of changing the flow environment on the tail surface. Akhtar and colleagues, using kinematic data from bluegill sunfish, showed through computational fluid dynamic calculations that the dorsal fin wake can substantially enhance thrust produced by the tail, if it is phased correctly (Akhtar et al., 2007). As the dorsal fin wake encounters the tail, it causes enhanced separation of the tail leading edge vortex, increasing the low pressure region on the tail surface during the time that the caudal fin is angled forward. This increases thrust. In these simulations,
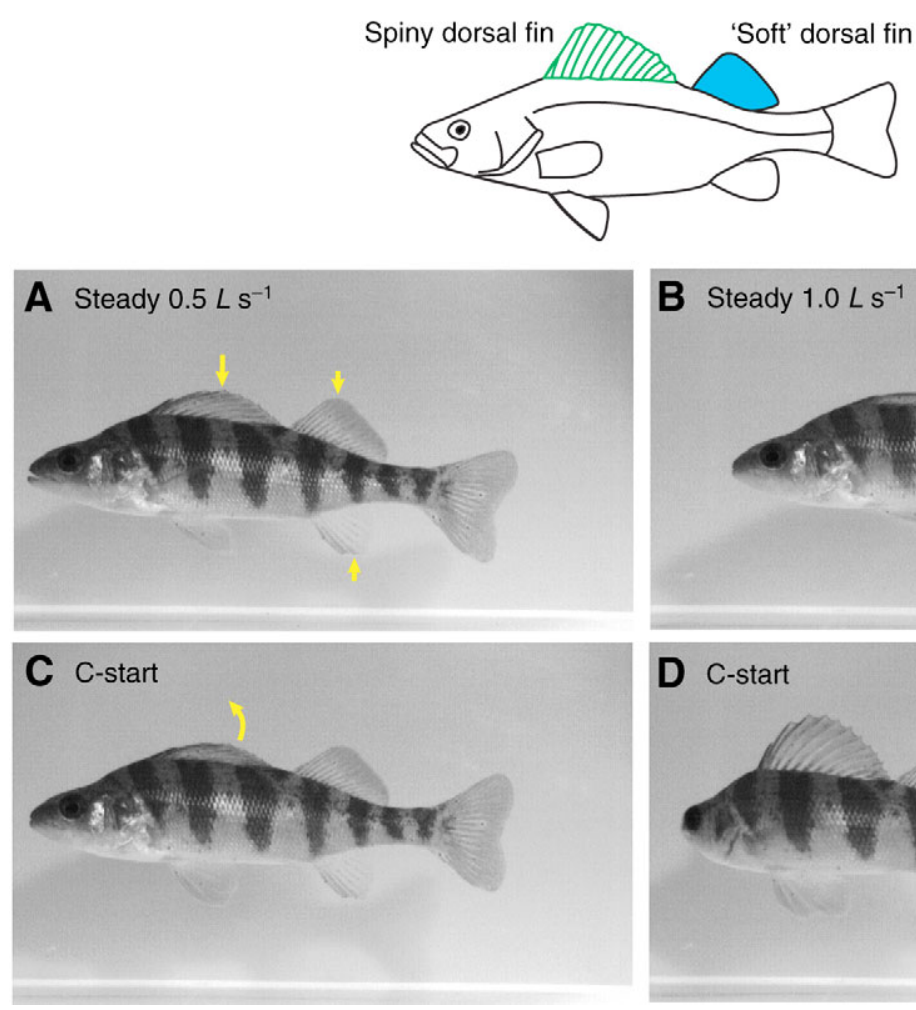

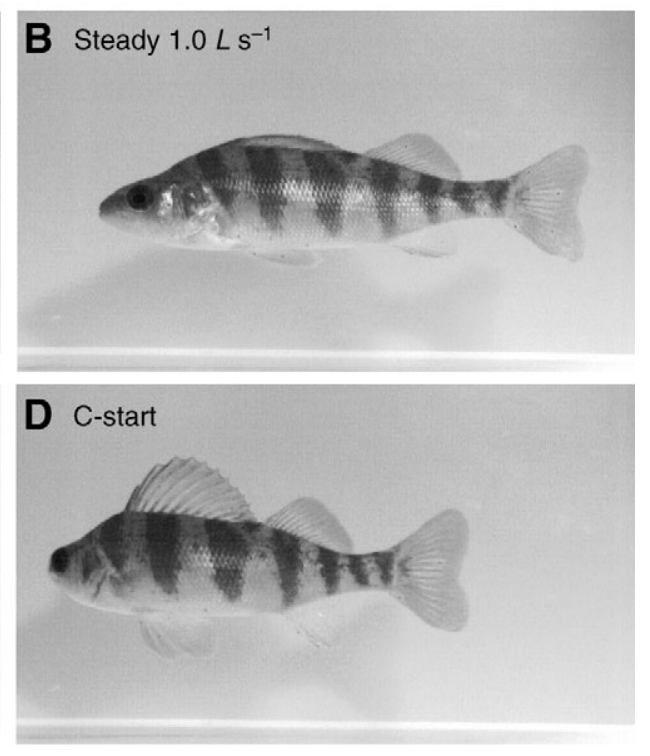

Fig. 9. Locomotion in yellow perch (Perca flavescens, $16.5 \mathrm{~cm}$ total length, $L)$ to illustrate the function of the dorsal fin during steady swimming and a c-start escape response. Yellow perch have a dorsal fin with distinct spiny and 'soft' portions, in contrast to bluegill sunfish (e.g. Fig. 8). $(A, B)$ Frames from a high-speed video $(250 \mathrm{~Hz})$ of steady swimming at two speeds ( 0.5 and $1.0 \mathrm{~L} \mathrm{~s}^{-1}$ ). Note that the height of the anterior spiny and posterior soft portions of the dorsal fin as well as the anal fin decrease with increasing swimming speed (yellow arrows). (C) Dorsal fin conformation just prior to a c-start; (D) fin position toward the end of stage 1 of the cstart. The spiny portion of the dorsal fin is erected during stage 1 (yellow arrow), but the soft dorsal and anal fin show little change in area. 
the tail thrust coefficient nearly doubled and the efficiency increased by $52 \%$ compared with the tail alone in the absence of a dorsal fin wake (Akhtar et al., 2007).

In rainbow trout, the dorsal fin generates little additional thrust, but strong side forces: the side:posterior force ratio is almost 6:1 at a swimming speed of $1.0 \mathrm{~L} \mathrm{~s}^{-1}$. The rainbow trout dorsal fin wake consists of a chain of vortices that move toward the tail, and the caudal fin oscillates through the centers of these shed vortices (Drucker and Lauder, 2005).

In both sunfish and trout, the dorsal fin wake changes significantly with speed, but the changes differ considerably between the species. In sunfish, the dorsal fin oscillates with very low amplitude at slow swimming speeds, and increases both movement and force production as speed increases. In contrast, data on the rainbow trout dorsal fin show that amplitude and lateral force generation are maximal at low swimming speeds, and decrease as speed increases. The differences between species may reflect the different body shapes, and hence differences in the ability of each species to maintain stability. Further comparative hydrodynamic data will allow a quantitative, mechanistic examination of how body shape and median fin placement affect stability among species.

Many bony fishes possess more than one dorsal fin, and the major group of teleost fishes known as the Acanthopterygii (Lauder and Liem, 1983; Nelson, 2006) possess a dorsal fin with an anterior spiny portion and a posterior soft-rayed region. The soft-rayed region of the fin possesses inclinator muscles which allow active side-to-side motion (Jayne et al., 1996) of the typical bony fish fin rays supporting this fin (Alben et al., 2007), while the spiny region of the fin possesses only erector and depressor muscles acting on rigid spines, but no inclinators (Winterbottom, 1974). The spiny dorsal fin thus can move laterally only in concert with body motion. In bluegill sunfish these two dorsal fin regions are attached (e.g. Fig. 8), but in many species the spiny region is separate from the soft-rayed region.

Yellow perch (Perca flavescens) possess distinct soft and spiny regions of the dorsal fin, and these two fin regions can function differently during locomotion (Fig. 9). During steady swimming, the height of both portions of the dorsal fin decreases as swimming speed increases, while during a c-start, the spiny region of the dorsal fin is erected while the soft portion of the fin changes little in height (Fig. 9). During steady swimming, the spiny dorsal fin sheds a drag wake that generates a shear layer that passes back over the soft dorsal fin (Fig. 7); no lateral momentum is evident in the wake of the spiny dorsal fin. The soft dorsal fin of perch also sheds a drag wake, but with significant lateral momentum that passes back and is encountered by the tail during steady locomotion (Fig. 7).

\section{Maneuvering}

In steady swimming, the forces and moments from all of the fins must balance over the course of a fin beat. When they do not balance, the velocity of a fish changes, which is the most general definition of maneuvering. In three dimensions, maneuvering can occur along three rotational axes: left or right turns (yaw), rolling along the fish's long axis, and pitching the snout upwards or downwards. Maneuvers often involve 3D movements of the fins and body (Fig. 3) (Standen and Lauder, 2005). For example, during yaw turns, the dorsal and anal fins tend to oscillate asymmetrically with a series of large excursions to one side of the body, often pausing at maximum excursion between oscillations. This asymmetrical behavior results in the formation of large lateral jets by dorsal and anal fins and a lateral and often dorso-ventral displacement of the fish's body (Fig. 10).

Lateral momentum jets also form around the separate spiny and soft portions of the dorsal fin in yellow perch, and the resulting wake flows with two dorsal fins and the caudal fin during a maneuver can be complex (Fig.9). By the end of a yawing maneuver in this species, three distinct vortex rings are generated, with multiple lateral momentum jets formed both by separation from the rigid trailing edge of the spiny dorsal fin and by active movement of the soft dorsal and caudal fin.

The timing of maximum circulation and resultant jet peak velocity from median fins do not always balance (Standen and

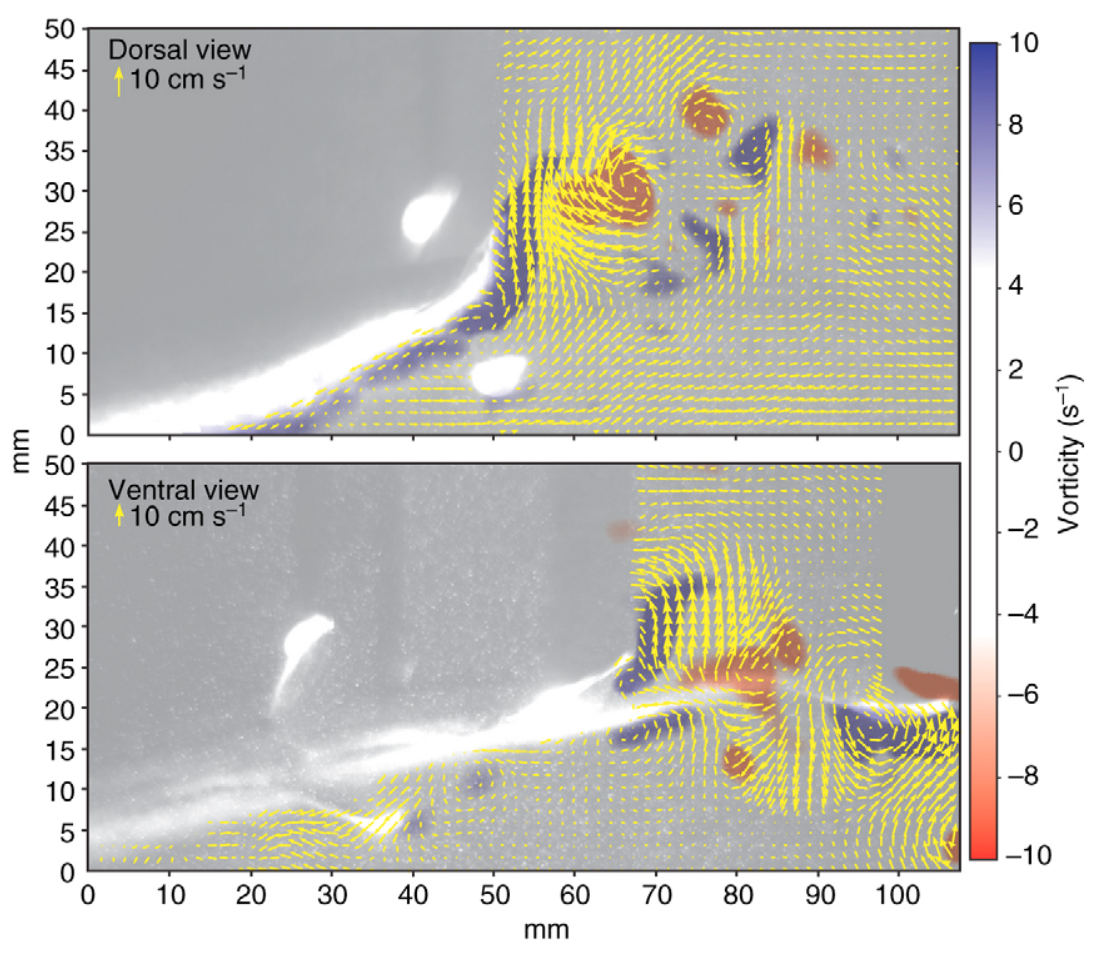

Fig. 10. Trout yawing maneuver at $0.5 L^{-1}$. The fish is maneuvering away from the top of each image. The dorsal view is taken while the dorsal fin pauses at maximum excursion, showing a large shear layer along the left side of the fin and the formation of a strong lateral jet. The ventral view is taken just after the anal fin begins to return from maximum excursion; again a large shear layer is seen on the left side of the fin also accompanied by a large lateral jet. During yawing maneuvers, contralateral jet formation is typically weak, causing an imbalance in force production and moving the fish's body laterally away from the strong jet side of the fish. Shadows where vectors were not calculated represent regions where the laser light sheet was blocked by the fish body or fins (after Standen and Lauder, 2007). 
Lauder, 2007), indicating that fish can fine tune force production around the body to control body position. Understanding the mechanics of maneuvers will require examination of the contributions of all of the fins and their 3D shapes and motions.

\section{Conclusions and prospectus}

Fish are 3D. Despite this fact, considerable progress has been made over the past 30 years in understanding the basic dynamics of aquatic locomotion using 2D models (e.g. Lighthill, 1975; Schultz and Webb, 2002). 2D simplifications have allowed progress in understanding the fundamental mechanisms of thrust generation in fishes (reviewed in Lauder and Tytell, 2006), and have permitted the use of relatively less-complex technology to provide data for testing models and understanding the first-order diversity of fish movement patterns. Using only a single high-speed camera, one can readily obtain body outlines for swimming fishes, quantify how patterns of body bending change with speed, and relate body bending to muscle electrical activity and strain (Shadwick and Gemballa, 2006).

But quantifying the motion of the fish body, fins and water in three dimensions is a more challenging proposition. Fortunately, technical developments in recent years are increasingly making the move to three dimensions feasible for experimentalists (Lauder, 2006; Lauder and Tytell, 2006), and increases in computing power and the sophistication of computational algorithms have made 3D computation of fluid flows more practical also (Mittal, 2004; Mittal et al., 2006; Mittal and Iaccarino, 2005). On the experimental side, the use of multiple high-speed cameras to provide a variety of views of complexly deforming fish fins is now practical (Lauder et al., 2006; Standen and Lauder, 2005), and the use of stereo particle image velocimetry, multiple simultaneous light sheets, transverse light sheet orientations and scanning PIV all make 3D reconstruction of fluid flows feasible (Burgmann et al., 2006; Nauen and Lauder, 2002b; Standen and Lauder, 2007; Tytell, 2006). The coming marriage of 3D experimental and computational approaches promises exciting progress toward understanding fish locomotor dynamics.

This work was supported by NIH grant 5 F32 NS054367 to E.D.T., by NSF grant IBN0316675 to G.V.L., and by ONR-MURI grant N00014-03-1-0897, monitored by Dr Thomas McKenna and initiated by Dr Promode Bandyopadhyay. We thank Drs Rajat Mittal and Promode Bandyopadhyay for many helpful discussions on bioinspired propulsion. Video data presented herein on yellow perch fin function were collected in collaboration with Dr Eliot Drucker, and we are grateful for his permission to use those data here. Brooke Flammang kindly provided data on bluegill sunfish tail function during maneuvering.

\section{References}

Abbott, E. A. (1899). Flatland: A Romance of Many Dimensions. Boston: Little, Brown, and Co.

Akhtar, I., Mittal, R., Lauder, G. and Drucker, E. (2007). Hydrodynamics of a biologically inspired tandem flapping foil configuration. Theor. Comput. Fluid Dyn. 21, 155-170.

Alben, S., Madden, P. G. A. and Lauder, G. V. (2007). The mechanics of active finshape control in ray-finned fishes. J. R. Soc. Interface 4, 243-256.

Anderson, E. J., McGillis, W. R. and Grosenbaugh, M. A. (2001). The boundary layer of swimming fish. J. Exp. Biol. 204, 81-102.

Bainbridge, R. (1963). Caudal fin and body movements in the propulsion of some fish. J. Exp. Biol. 40, 23-56.

Block, B. A. and Stevens, E. D. (2001). Tuna: Physiology, Ecology, and Evolution: Fish Physiology, Vol. 19 (ed. W. S. Hoar, D. J. Randall and A. P. Farrell). San Diego: Academic Press.

Breder, C. M. (1926). The locomotion of fishes. Zoologica 4, 159-297.

Burgmann, S., Brucker, C. and Schroder, W. (2006). Scanning PIV measurements of a laminar separation bubble. Exp. Fluids 41, 319-326.

Drucker, E. G. and Lauder, G. V. (1999). Locomotor forces on a swimming fish: three-dimensional vortex wake dynamics quantified using digital particle image velocimetry. J. Exp. Biol. 202, 2393-2412.

Drucker, E. G. and Lauder, G. V. (2001). Locomotor function of the dorsal fin in teleost fishes: experimental analysis of wake forces in sunfish. J. Exp. Biol. 204 2943-2958.
Drucker, E. G. and Lauder, G. V. (2005). Locomotor function of the dorsal fin in rainbow trout: kinematic patterns and hydrodynamic forces. J. Exp. Biol. 208, 4479-4494.

Gillis, G. B. (1998). Environmental effects on undulatory locomotion in the American eel Anguilla rostrata: kinematics in water and on land. J. Exp. Biol. 201, 949-961.

Gray, J. (1933). Studies in animal locomotion. I. The movement of fish with special reference to the eel. J. Exp. Biol. 10, 88-104.

Jayne, B. C. and Lauder, G. V. (1995). Speed effects on midline kinematics during steady undulatory swimming of largemouth bass, Micropterus salmoides. J. Exp. Biol. 198, 585-602.

Jayne, B. C., Lozada, A. F. and Lauder, G. V. (1996). Function of the dorsal fin in bluegill sunfish: motor patterns during four distinct locomotor behaviors. J. Morphol. 228, 307-326.

Lauder, G. V. (2000). Function of the caudal fin during locomotion in fishes: kinematics, flow visualization, and evolutionary patterns. Am. Zool. 40, 101-122.

Lauder, G. V. (2006). Locomotion. In The Physiology of Fishes (ed. D. H. Evans and J. B. Clairborne), pp. 3-46. Boca Raton: Taylor and Francis.

Lauder, G. V. and Liem, K. F. (1983). The evolution and interrelationships of the actinopterygian fishes. Bull. Mus. Comp. Zool. Harvard 150, 95-197.

Lauder, G. V. and Tytell, E. D. (2004). Three Gray classics on the biomechanics of animal movement. J. Exp. Biol. 207, 1597-1599.

Lauder, G. V. and Tytell, E. D. (2006). Hydrodynamics of undulatory propulsion. In Fish Biomechanics (ed. R. E. Shadwick and G. V. Lauder), pp. 425-468. San Diego: Academic Press.

Lauder, G. V., Madden, P. G. A., Mittal, R., Dong, H. and Bozkurttas, M. (2006). Locomotion with flexible propulsors: I. Experimental analysis of pectoral fin swimming in sunfish. Bioinspir. Biomim. 1, S25.

Lighthill, J. (1960). Note on the swimming of slender fish. J. Fluid Mech. 9, 305-317.

Lighthill, J. (1970). Aquatic animal propulsion of high hydromechanical efficiency. J. Fluid Mech. 44, 265-301.

Lighthill, J. (1971). Large-amplitude elongated-body theory of fish locomotion. Proc R. Soc. Lond. B Biol. Sci. 179, 125-138.

Lighthill, J. (1975). Mathematical Biofluiddynamics. Philadelphia: Society for Industria and Applied Mathematics.

Marshall, N. B. (1971). Explorations in the Life of Fishes. Cambridge, MA: Harvard University Press.

Mittal, R. (2004). Computational modeling in biohydrodynamics: trends, challenges, and recent advances. IEEE J. Oceanic Eng. 29, 595-604.

Mittal, R. and laccarino, G. (2005). Immersed boundary methods. Annu. Rev. Fluid Mech. 37, 239-261.

Mittal, R., Dong, H., Bozkurttas, M., Lauder, G. V. and Madden, P. G. A. (2006). Locomotion with flexible propulsors. Il. Computational modeling of pectoral fin swimming in sunfish. Bioinspir. Biomim. 1, S35.

Müller, U. K., van den Heuvel, B.-L. E., Stamhuis, E. J. and Videler, J. J. (1997) Fish foot prints: morphology and energetics of the wake behind a continuously swimming mullet (Chelon labrosus Risso). J. Exp. Biol. 200, 2893-2906.

Müller, U. K., Smit, J., Stamhuis, E. J. and Videler, J. J. (2001). How the body contributes to the wake in undulatory fish swimming: flow fields of a swimming eel (Anguilla anguilla). J. Exp. Biol. 204, 2751-2762.

Nauen, J. C. and Lauder, G. V. (2001). Locomotion in scombrid fishes: visualization of flow around the caudal peduncle and finlets of the chub mackerel Scomber japonicus. J. Exp. Biol. 204, 2251-2263.

Nauen, J. C. and Lauder, G. V. (2002a). Hydrodynamics of caudal fin locomotion by chub mackerel, Scomber japonicus (Scombridae). J. Exp. Biol. 205, 1709-1724.

Nauen, J. C. and Lauder, G. V. (2002b). Quantification of the wake of rainbow trout (Oncorhynchus mykiss) using three-dimensional stereoscopic digital particle image velocimetry. J. Exp. Biol. 205, 3271-3279.

Nelson, J. S. (2006). Fishes of the World. Hoboken, NJ: John Wiley.

Schultz, W. W. and Webb, P. W. (2002). Power requirements of swimming: do new methods resolve old questions? Integr. Comp. Biol. 42, 1018-1025.

Shadwick, R. E. and Gemballa, S. (2006). Structure, kinematics, and muscle dynamics in undulatory swimming. In Fish Biomechanics: Fish Physiology. Vol. 23 (ed. R. E. Shadwick and G. V. Lauder), pp. 241-280. San Diego: Academic Press.

Standen, E. M. and Lauder, G. V. (2005). Dorsal and anal fin function in bluegill sunfish (Lepomis macrochirus): three-dimensional kinematics during propulsion and maneuvering. J. Exp. Biol. 208, 2753-2763.

Standen, E. M. and Lauder, G. V. (2007). Hydrodynamic function of dorsal and anal fins in brook trout (Salvelinus fontinalis). J. Exp. Biol. 210, 325-339.

Taylor, G. I. (1952). Analysis of the swimming of long and narrow animals. Proc. R. Soc. Lond. A Math. Phys. Sci. 214, 158-183.

Tytell, E. D. (2004). The hydrodynamics of eel swimming. II. Effect of swimming speed. J. Exp. Biol. 207, 3265-3279.

Tytell, E. D. (2006). Median fin function in bluegill sunfish, Lepomis macrochirus: streamwise vortex structure during steady swimming. J. Exp. Biol. 209, 1516-1534.

Tytell, E. D. and Lauder, G. V. (2004). The hydrodynamics of eel swimming. I. Wake structure. J. Exp. Biol. 207, 1825-1841.

van Ginneken, V., Antonissen, E., Muller, U. K., Booms, R., Eding, E., Verreth, J. and van den Thillart, G. (2005). Eel migration to the Sargasso: remarkably high swimming efficiency and low energy costs. J. Exp. Biol. 208, 1329-1335.

Weihs, D. (1972). A hydrodynamical analysis of fish turning manoevers. Proc. R. Soc Lond. B Biol. Sci. 182, 59-72.

Willert, C. E. and Gharib, M. (1991). Digital particle image velocimetry. Exp. Fluids 10, 181-193

Winterbottom, R. (1974). A descriptive synonymy of the striated muscles of the Teleostei. Proc. Acad. Nat. Sci. Philadelphia 125, 225-317.

Wu, T. Y. (1971). Hydromechanics of swimming propulsion. Part 1. Swimming of a two-dimensional flexible plate at variable forward speeds in an inviscid fluid. J. Fluid Mech. 46, 337-355.

Zhu, Q., Wolfgang, M. J., Yue, D. K. P. and Triantafyllou, M. S. (2002). Threedimensional flow structures and vorticity control in fish-like swimming. J. Fluid Mech $468,1-28$. 